\title{
Hybrid
}

Revue des arts et médiations humaines

$4 \mid 2017$

Malaise dans la représentation

\section{La contagion des images : voix enchâssées et dispositifs sensoriels dans un rituel chamanique amérindien}

Andrea-Luz Gutierrez Choquevilca

\section{(2) OpenEdition \\ Journals}

Édition électronique

URL : https://journals.openedition.org/hybrid/799

DOI : 10.4000/hybrid.799

ISSN : 2276-3538

Traduction(s) :

The contagion of images: embedded voices and sensory apparatuses in an Amerindian shamanic ritual - URL : https://journals.openedition.org/hybrid/807 [en]

Éditeur

Presses universitaires de Vincennes

Référence électronique

Andrea-Luz Gutierrez Choquevilca, « La contagion des images : voix enchâssées et dispositifs sensoriels dans un rituel chamanique amérindien », Hybrid [En ligne], 4 | 2017, mis en ligne le 04 septembre 2017, consulté le 13 avril 2022. URL : http://journals.openedition.org/hybrid/799; DOI : https://doi.org/10.4000/hybrid.799

Ce document a été généré automatiquement le 13 avril 2022.

Revue Hybrid 


\title{
La contagion des images : voix enchâssées et dispositifs sensoriels dans un rituel chamanique amérindien
}

\author{
Andrea-Luz Gutierrez Choquevilca
}

\section{Introduction}

1 Cet article propose d'interroger à partir d'un exemple amazonien l'un des enjeux centraux de l'énonciation rituelle chamanique : l'opération de composition-traduction qui s'impose au spécialiste rituel pour «représenter» les invisibles au cours de sa performance, et les dispositifs sensoriels qu'elle implique. Par quels moyens linguistiques, gestuels ou sensoriels le chamane yachak parvient-il à résoudre le déficit de visibilité des êtres convoqués sur la scène des performances rituelles ? Comment des idées aussi abstraites et difficiles à circonscrire que celles d' "esprit ", de « démon » ou $d^{\prime}$ ' âme ${ }^{1}$ » viennent-elles trouver une résonance dans l'expérience la plus intime des peuples amérindiens? Une méthode pour aborder cette question s'est révélée particulièrement féconde en anthropologie ${ }^{2}$. Elle consiste à interroger à partir de matériaux ethnographiques les moyens cognitifs, discursifs ou sensoriels employés lors des performances rituelles pour figurer ces entités et communiquer à leur sujet ou avec elles. L'attention se déplace de la description d'une vision du monde particulière vers les dispositifs empiriques d' "élaboration de ces mondes", ways of worldmaking pour emprunter une formule chère à Nelson Goodman ${ }^{3}$. Ces dispositifs, plutôt qu'ils ne dévoilent des savoirs fossilisés, constituent des performances culturelles à certains égards comparables à celles déployées dans ce que nous avons coutume d'appeler « œuvre d'art » en Occident ${ }^{4}$. Loin d'augmenter la distance qui sépare les performances artistiques et rituelles à proprement parler, l'anthropologie du chamanisme souligne au contraire ce que les mécanismes de dévoilement et d'occultation des images ont en 
commun, parce qu'ils se fondent sur une aptitude humaine fondamentale, la communication.

L'image rituelle s'entend ici comme le support d'une appréhension perceptive (visuelle, sonore, olfactive, etc.) et cognitive : on parlera dans ce cas volontiers d'image mentale. Elle donne lieu à une attribution de subjectivité déplacée par l'expert rituel qui la manipule : l'évocation de la petite couleuvre taruna est, de son point de vue, l'esprit présenté à l'assistance au moment de l'acte thérapeutique, quand bien même cet événement resterait partiellement inintelligible à l'auditoire agenouillé autour du malade. L'une des conséquences de cette croyance commune est qu'un rituel thérapeutique réussi est un rituel dans lequel les images font l'objet d'une contagion entre les participants : le patient imagine, voit, sent, perçoit «quelque chose » qu'il peine à décrire avec des mots. Ces images font l'objet d'un déchiffrement variable de la part des acteurs rituels, et s'inscrivent de ce fait dans une forme de communication spéciale. Ce sont les ressorts de ces actes de communication qui nous intéressent ici.

3 À partir d'un retour critique sur la notion d'image agissante et celle d'«efficacité symbolique » chère à Lévi-Strauss, nous examinerons les conditions perceptives et cognitives de l'adhésion au rituel amérindien. Cette ligne d'analyse se fonde sur une ethnographie détaillée des rituels chamaniques des sociétés Runa d'Amazonie péruvienne ${ }^{5}$ et sur une analyse pragmatique des régimes discursifs de figuration des acteurs du rituel. Qu'advient-il lorsque l'enjeu du rituel consiste en l'obviation pure et simple des règles de la communication ordinaire? La particularité du rite chamanique est que le spécialiste semble seul à détenir la clef des jeux lexicaux et des dispositifs sensoriels qu'il met en place. Une certaine perplexité contamine l'auditoire, le patient et le néophyte. Le paradigme occidental de la communication, défini par les sciences du langage autour de la notion d'intention signifiante et de pertinence, se voit ici subverti au profit d'une performance fondée sur la multiplication des voix, des points de vue et des interprétations possibles de l'image. L'ethnographie du rituel thérapeutique permet d'examiner l'hypothèse que c'est précisément, dans ce contexte, l'aspect lacunaire du signe - la transgression des règles de la communication ordinaire et le caractère partiel du déchiffrement - qui confère à l'image toute sa force.

\section{L'image efficace : Guillermo Haya et « La Voie de Muu- Igala »}

4 Les ethnographies consacrées aux systèmes de pensée et de croyances chamaniques révèlent que les traditions amérindiennes sont tout autant orales qu' i iconographiques ». L'étude des chants énoncés par les experts rituels amazoniens met en lumière un déroulement d'images dont la scénographie - chantée, vue, sentie ou ressentie - fait coïncider un acte de figuration et un acte thérapeutique, c'est-à-dire une action de transformation du vivant, des corps, de la matière. "Avec mes chants ikara puissants nous faisons revenir les petites âmes. Mes ikara guérissent et tuent. Ne les entends-tu pas qui sifflent sur ta tête, lorsque je soigne? » me demandait un chamane runa appelé Yana Shunku, «foie noir». Difficile de ne pas y voir une allusion explicite au fait que sa performance sonore excède ce qu'elle donne à percevoir concrètement, quelques phrases sibyllines murmurées entre deux bouffées de fumée lâchées sur un corps silencieux. C'est de l'image agissante dont il est ici question. Outre la dimension performative du discours, il est important de rappeler combien l'énonciation de ces 
chants est étroitement liée à des performances puisant ailleurs, dans d'autres registres de savoirs et d'expériences perceptives : la transmission du souffle, la fumée de tabac, le son de la voix du chamane transformée en sifflement ou en musique, le bruissement $\mathrm{du}$ hochet de feuilles effleurant la peau du malade étendu sur la natte... Sous quels régimes de visibilité le chamane amérindien, celui que l'on nomme yachak, «celui qui sait » en langue quechua, dévoile-t-il les êtres convoqués sur la scène rituelle : esprits, démons, animaux ou entités hybrides? Comment expliquer la singulière efficace attribuée à la poétique rituelle?

5 Tournons-nous vers le texte où vient puiser Claude Lévi-Strauss pour aborder précisément cette question, texte dont l'auteur aura été dramatiquement oublié de tous. C'est en effet un indien Cuna nommé Guillermo Haya qui recueillait à Panama à la fin des années 1940 un chant énoncé par un chamane de son village auprès d'une parturiente, à l'occasion d'un accouchement difficile : «La Voie de Muu-Igala». Ce texte de plume indigène, rare pour l'époque, transcrit par l'auteur dans sa propre langue cuna et traduit en espagnol, fut envoyé à l'ethnographe suédois Erland Nordenskiöld, puis publié après la mort de ce dernier par ses successeurs Nils M. Holmer et Henry Wassen à Göteborg en 1947. L'engouement qu'éprouva Lévi-Strauss à la lecture de ce chant lui inspira en 1949 l'écriture d'un article intitulé "L'efficacité symbolique » qui deviendra plus tard un chapitre d'Anthropologie structurale (1958). Cet article séminal et controversé propose une nouvelle exégèse des paroles chamaniques cuna à travers le prisme de la "performance ». Il est animé par un désir de comprendre ce qui se joue lorsque ce chant est énoncé par la parole vive du chamane, au cœur du rituel. Claude Lévi-Strauss y décompose l'analogie qui s'établit termes à termes entre le corps douloureux de la parturiente indienne et les images évoquées par les paroles du chamane. Ici, dit-il, « le chant constitue une manipulation psychologique de l'organe de la malade, et cette manipulation est la guérison attendue ${ }^{6} »$. Immergé dans le récit de Haya dont il tire toute la substance ethnographique, Lévi-Strauss observe l'oscillation dans le texte entre thèmes mythiques et physiologiques. La croyance s'établit « comme s'il s'agissait d'abolir, dans l'esprit de la malade la distinction qui les sépare, et de rendre impossible la différenciation de leurs attributs respectifs ${ }^{7}$ ». Le chamane Cuna exhorte ses esprits protecteurs figurés par des statuettes sculptées en bois appelées nuchu:

Le malade git dans son hamac, devant vous,

Son blanc tissu est allongé,

Son blanc tissu remue doucement.

Le faible corps de la malade est étendu ;

Quand ils éclairent le chemin de Muu, celui-ci ruisselle, de sang! (v. 84-90) ${ }^{8}$.

Dans cet obscur « chemin de Muu-Igala » ensanglanté par l'accouchement difficile, la glose rituelle rend visibles les "vêtements magiques" à la lueur desquels les esprits protecteurs nuchu pourront reconnaitre le corps de la malade, afin d'en obtenir la guérison. Il est remarquable ici qu'aucun des organes ou des entités en présence, qu'il s'agisse du patient, du chamane ou des esprits protecteurs, ne soit désigné par un terme familier explicite ou univoque. Tout se passe au contraire comme si, la primauté étant accordée à la polysémie et au glissement sémantique, il fallait soigneusement éviter la référence directe aux objets, aux corps, au profit de la création d'images multiples, évanescentes et incomplètes. Le vagin de la parturiente est ainsi désigné comme le "sentier de Muu-Igala », les douleurs exposées sous la forme figurée de monstres ou 
d'« animaux accroissant les maux de la femme en travail ", d'après Guillermo Haya. C'est :

Oncle Alligator Tiikwalele, au corps luisant, qui remue ses luisantes nageoires, dont les nageoires envahissent la place, repoussent tout, entraînent tout; Nele Ki (k) kirpanalele, la Pieuvre, dont les tentacules gluantes sortent et rentrent alternativement; et bien d'autres encore: Celui-dont-le-chapeau-est-mou, Celuidont-le-chapeau-est-rouge, Celui-dont-le-chapeau-est-multicolore; et les animaux gardiens: le Tigre-noir, l'Animal-rouge, l'Animal-bicolore, l'Animal-couleur-depoussière ; chacun attaché par une chaîne de fer, langue pendante, langue sortante, bavant, écumant, la queue flamboyante, les dents menaçantes et déchirant tout, « tout comme du sang tout rouge » (v. 253-298) ${ }^{9}$.

7 Théâtre redoutable de douleurs incarnées tel un Enfer à la Jérôme Bosch, le chant déverse dans un flux continu des images qui semblent n'avoir d'équivalent que dans le mythe, images que la parturiente voit ici présentées sur le «lieu» de sa propre expérience corporelle. De quels outils l'anthropologie dispose-t-elle pour ausculter ce mouvement de contagion des images parmi les participants lors du rituel chamanique? Lévi-Strauss soutient, dans ce texte célèbre consacré à l'« efficacité » rituelle, que la question qu'il nous faut résoudre s'énonce de la manière suivante : peut-on formuler l'informulable? Comment le néophyte et le patient "profane " parviennent-ils à s'intégrer dans un dispositif de communication qui les transcende largement, au point de fonder leur croyance sur un ensemble ténu d'indices improbables? L'adhésion au rituel et la création d'un terrain d'entente ${ }^{10}$ commun entre experts, profanes et invisibles est en effet contemporaine de la production d'une constellation d'indices, d'icones et de signes - souvent l'image articulée avec la parole - qui contribuent à atténuer l'opacité de la performance et à instaurer les conditions cognitives et sensorielles d'un partage. "Le shaman, expliquait Lévi-Strauss, fournit à sa malade un langage, dans lequel peuvent s'exprimer immédiatement des états informulés, et autrement informulables ${ }^{11}$.»

D'importantes critiques ont été adressées à l'interprétation que propose Lévi-Strauss de la formulation de ces "mondes possibles» par le spécialiste rituel amérindien, notamment à l'interprétation symbolique qu'il en donne ${ }^{12}$. Ces réactions soulignent à la fois l'intérêt intrinsèque de l'objet choisi et la difficulté analytique à laquelle l'ethnologue se voyait confronté. Là où Lévi-Strauss pensait déceler dans cette dialectique entre l'image du mythe et celle des corps un rapprochement heureux avec la psychanalyse $^{13}$, le lecteur ne rencontre hélas aucun élément permettant de comprendre dans le temps et sur le lieu de la performance elle-même, la contagion de telles croyances parmi les acteurs du rituel. L'explication symbolique se heurte au constat que la plupart des paroles énoncées par le chamane demeurent souvent irréductiblement opaques et peu intelligibles au néophyte, que l'action rituelle est la plupart du temps aussi non verbale, qu'elle est médiatisée in loci verbi par des objets... Pourquoi, en l'occurrence, se met ici en place une pratique discursive "spéciale", formalisée par des contraintes énonciatives spécifiques: le parallélisme, des métaphores opaques, des listes de noms d'esprits, autant de techniques rhétoriques classiques bien connues dans la littérature ${ }^{14}$ ?

La difficulté à ethnographier un tel dispositif tient à sa nature: irréductible à la communication langagière bien que passant nécessairement par celle-ci, il affleure à travers les gestes et relations corporelles entre les participants du rituel, à travers les substances transmises, les visions des initiés mises en paroles, confiées aux invisibles. 
Une véritable opération de traduction est ici en jeu, opérant sur ces trois niveaux relevés par Jakobson : intralinguistique, interlinguistique, et intersémiotique, épousant les contours des relations dissidentes et complices entre le langage, les sens et la pensé ${ }^{15}$. Il n'est pas nécessaire, une telle voie paraît aujourd'hui galvaudée tant le projet structuraliste diffère par son enjeu, d'opposer de façon diamétrale l'approche lévi-straussienne et celle qui vise à rendre compte d'une pragmatique des actes de communication rituelle. En effet, Lévi-Strauss pressentait lui-même avec acuité la difficulté à articuler la question de la performance avec celle de la dimension formelle de l'action rituelle et le type de communication singulier qui s'y déroule. Conscient que l'action rituelle ne peut se résumer à la parole énoncée et encore moins au script qu'en donne le mythe, il ouvrait une ligne de recherche novatrice dans le final des Mythologiques en soulignant qu'il serait plus productif de s'abstenir de regarder «ce que disent ces paroles rituelles pour voir comment elles disent ${ }^{16} »$. C'est autre chose qu'une théorie de la référence, centrée sur la relation nécessaire unissant le signe et l'objet qu'il dénote dans le monde ${ }^{17}$, qui se voit engagée dans l'analyse de l'action rituelle. C'est une pragmatique sensible aux intentions dissimulées derrière les mots ou les gestes, aux savoirs d'arrière-plan et aux implicites de l'action, qui meuvent les humains et leurs partenaires invisibles.

\section{L'indétermination de la référence ou l'énigme rituelle}

10 Que nous enseigne l'ethnographie contemporaine des peuples amérindiens sur l'efficacité accordée aux images rituelles? Il nous faut revenir sur la biographie d'un être peu ordinaire, le chamane yachak, "celui qui sait", pour éclairer sa fonction d'opérateur de visibilité et d'occultation dans le théâtre rituel. Chez les peuples quechua d'Amazonie, le titre d'expert rituel vient couronner une longue et pénible initiation, consistant en l'expérience d'une mort rituelle sasina et d'une dissolution du corps du chamane au contact avec ses esprits initiateurs. Transi par la faim, en raison de la prescription d'un jeûne drastique, l'impétrant est soumis à l'exercice laborieux des visions, car l'ensemble des chants ikara, qui définissent son aptitude à guérir, lui sont transmis par des êtres oniriques, animaux ou esprits rencontrés en rêve. La mémorisation d'un stock de chants thérapeutiques est l'enjeu principal de son initiation. Toutefois cette technique de mémorisation a pour particularité qu'elle est décrite par les novices comme relevant essentiellement d'une expérience corporelle non verbale: une expérience sensorielle générée par l'ingestion de substances inaccoutumées ou toxiques (breuvages végétaux, larves, insectes, petites pierres), la transformation de sa voix par la pratique de l'imitation de voix animales: celle du piauhau hurleur, du pécari ou des esprits aquatiques ${ }^{18}$. Ces éléments figurent comme autant d'indices sensibles matérialisant les relations que l'initié aura tissées avec les entités invisibles, dont il devient l'exclusif dépositaire et porte-parole aux yeux des profanes. Le corps du chamane est figuré à travers ces témoignages comme un corps chimérique, empli de fléchettes hématophages wiruti, extraites une à une du corps de son maître, au cours de son apprentissage. 


\begin{abstract}
Kay wiruti kallu kallu shina rikurin, ninchi "shun » chasna rikunayu kapayan. Kasha kasha kasha apillapas : kasna wiña' wiña' tukun.
\end{abstract}

Chupashpan surkushkani amun shiminmanta, rikuchihuni : chayta yachakunchi llawsawa supay wirutiwapas.
Ce dard [wiruti] ressemble à une sangsue kallu kallu, on l'appelle "shun », il ressemble à cela depuis les commencements des temps. C'est une épine tranchante, mais elle est tendre : et elle croît, elle ne cesse de croître.

En suçant [chupa-] je l'ai extrait de la bouche du maître : nous apprenons cela avec la bave [llawsa] et les dards des démons.

11 D'un point de vue cognitif, une telle articulation de la mémoire sémantique à une mémoire de type autobiographique est fondamentale, car elle vient ancrer la performance de l'action de guérison dans une expérience plus ancienne qui coïncide pour l'expert avec un détournement de la fonction traditionnelle assignée au langage et à la perception. Communiquer par des icones sonores peu ou prou inintelligibles, nourrir des êtres ou des objets qui sont à la fois au-dehors et au-dedans, ces petits "aïeux» ou esprits auxiliaires nommés supay wiruti, constituent des aptitudes graduellement acquises, venant conforter le sentiment qu'éprouve le chamane d'une étrangeté vécue dans l'intimité de son propre corps.

Mais si le discours portant sur l'apprentissage paraît minimiser le canal de l'oralité, il n'en fait pas moins surgir un paradoxe. Ce paradoxe réside dans l'association étroite entre le pouvoir de guérison et l'aptitude à maîtriser les techniques d'énonciation rituelle, qui se manifestent quant à elles toujours à travers des performances orales, plus exactement des chants. Les répertoires de chants chamaniques ikara constituent pour les peuples Runa de Haute Amazonie péruvienne une tradition ancienne, répandue sur une région vaste du Nord-Ouest amazonien. Le pouvoir de guérison demeure entièrement suspendu au "souffle" de la voix plutôt qu'à d'autres techniques, tel l'usage des pharmacopées, essentielles lors de l'initiation, mais peu efficaces lorsqu'il s'agit d'extraire les maux du corps des victimes de sorcellerie. Ce souffle thérapeutique est désigné par la racine verbale puku - dont la polysémie est caractéristique: elle se réfère tout autant au souffle du chasseur qui darde une fléchette empoisonnée au curare sur une proie arboricole, qu'à celui du chamane qui, pour sauver le malade ou l'ensorcelé, darde un « chant » ikara à l'intérieur de son corps, rendu poreux par l'usage du tabac et de la fumée. Autrement dit, guérir, c'est "souffler" ou entonner ces chants conçus comme des "projectiles" supay wiruti (fléchettes des esprits) contenus dans le corps du chamane.

13 À la façon de diplomates érudits, les chamanes runa circulent sur des territoires distants de leur région d'origine afin de transmettre leurs chants ikara aux peuples voisins : les peuples jivaro - Achuar, Aguaruna, les peuples de langue Shipibo (famille linguistique Pano). La transmission de ces chants repose sur l'emploi de formules stables dont la particularité est de combiner l'usage de règles syntaxiques strictes (le parallélisme, un marquage épistémique singulier, la technique de l'erotesis c'est-à-dire des questions rhétoriques) assorti d'importantes variations et d'innovations lexicales : chaque peuple emprunte à des langues voisines de nouveaux syntagmes (yora qui signifie "corps » en langue shipibo se voit ainsi transposé dans certains répertoires quechua), tandis que certains maîtres des chants innovent en créant des « noms » ou un code référentiel spécifique pour désigner de nouvelles catégories d'esprits. Chaque nom se réfère à une description imagée (sonore, visuelle, tactile, etc.) dont le contenu manifeste est livré lors d'une vision (muskuchi). Ainsi, l'onomatopée sisyawayttiii lexicalisation de l'icône sonore d'une voix non humaine - est devenue dans la région 
du Haut Pastaza péruvien le terme consacré pour désigner l'esprit de l'Anaconda lors du rituel thérapeutique, tandis que l'ophidien est nommé amarun dans la langue profane. L'esprit de l'aigrette est nommé par une métonymie, la mention d'un «vêtement couleur de cendre " (uchpa kamisayu), tandis que les dauphins roses des fleuves tropicaux, puissantes chimères aquatiques, sont nommés par la couleur rouge à laquelle s'ajoute un idéophone décrivant l'apparition sonore de l'esprit à la surface des eaux : puka-wayti $1^{19}$.

Ces jeux de substitution et de dénomination obliques sont légion dans les répertoires chamaniques amérindiens, au point que l'on soutient souvent que l'exercice d'une « langue tordue » - twisted language ${ }^{20}$ - serait l'un des fondements essentiels du discours chamanique et rituel.

Comment peut-on expliquer cette distorsion de la relation entre le signe et l'objet ou l'image mentale qu'il dénote? Un premier constat consiste à observer que dans le contexte de la prise en charge rituelle de la maladie, les modalités de la communication ordinaire se voient tout simplement dévoyées de leur fonction traditionnelle observée dans l'exercice quotidien du langage. En effet, les maximes de la communication introduites en philosophie du langage et en pragmatique par Herbert Paul Grice, considérées aujourd'hui comme centrales dans la pratique de la communication humaine, telles que le principe de coopération, les maximes de quantité (principe d'économie qui implique que l'information énoncée doit être nécessaire), de qualité (principe qui repose sur la sincérité du locuteur), de pertinence (qui suppose que l'information soit et suffisante et à-propos afin qu'un message puisse être correctement transmis ${ }^{21}$ se voient ici subverties au profit d'une performance fondée sur quelques traits formels constants et en apparence " absurdes ", pour emprunter une expression de Holmer et Wassen témoignant leur étonnement à la lecture de la voie de Muu Igala. Parmi ces traits figurent la répétition, la catachrèse, un usage immodéré de la périphrase, de stratégies de nominations fondées sur l'analogie ou la métonymie, l'adoption de règles syntaxiques épurées, la mise en place de listes de noms variables insérés dans un ordre paralléliste constant... Ces caractéristiques formelles ont été examinées au sein de la plupart des répertoires chamaniques amérindiens, des sociétés Suya du Brésil central aux Warao ou aux Piaroa du Venezuela, ou plus récemment chez les peuples Sharanahua et Marubo de langue Pano, au point qu'elles peuvent être envisagées comme des invariants révélant une forme de contrainte relativement stable de l'énonciation rituelle amérindienne ${ }^{22}$.

16 Ce chant récité par un chamane yachak chez les peuples Quechua d'Amazonie péruvienne, en offre l'épure. Plutôt qu'il ne "communique » un message, il pose en effet l'énigme - «Est-ce l'esprit qui rôde?» (supaychu) - dont on pressent que la formulation elle-même, par un jeu de mise en abyme, pourrait se décliner littéralement à l'infini, résumant le drame de la représentation : 
$1 \quad$ Sumpra-punta puri-k supay-chu Sumpra-uku puri-k supay-chu

Sumpra-washa purik supay-chu

Wayra-punta purik supaychu

$5 \quad$ Wayra-uku purik supaychu

Wayra-washa purik supaychu`

Tamya-punta purik supaychu tamya-uku purik supaychu

tamya-washa purik supaychu

10

Chawpi tuta-p' purik supaychu

Yaku-awa purik supaychu (...)

Purma-uku purik supaychu (...)

Kaspi-siki-pi tiyak supaychu (...)

Mishushillu-siki-pi shayak supaychu

15

Aychakama supaychu
Aux frontières de l'ombre est-ce l'esprit qui rôde? À l'intérieur de l'ombre est-ce l'esprit qui rôde?

Derrière l'ombre est-ce l'esprit qui rôde?

Aux frontières du vent est-ce l'esprit qui rôde?

À l'intérieur du vent est-ce l'esprit qui rôde?

Derrière le vent est-ce l'esprit qui rôde?

Aux frontières de la pluie est-ce l'esprit qui rôde? À l'intérieur de la pluie est-ce l'esprit qui rôde?

Derrière la pluie est ce l'esprit qui rôde?

Au milieu de la nuit est-ce l'esprit qui rôde?

Au dessus de la rivière est-ce l'esprit qui rôde?

À l'intérieur de la friche est-ce l'esprit qui rôde?

Au pied de l'arbre est-ce l'esprit qui est assis ?

Au pied de la liane mishu shillu (Uncaria tomentas, Rubiaceae) est-ce l'esprit qui se dresse?

Est-ce l'esprit Aychakama (esprit-maître des singes) ?

Plutôt qu'elle n'identifie un être singulier ou un lieu de capture de l'âme du malade, la parole rituelle entame un processus de subjectivation graduel, à partir d'une série d'indices topologiques traçant une espèce de carte mentale ${ }^{23}$. Le yachak inaugure une quête qui s'exprime par des métonymies déclinant les lieux de passage, l'habitat des esprits pathogènes où se trouve le reflet, l'âme capturée du patient. Le diagnostic coïncide alors avec la thérapie. D'une part, il s'agit pour le yachak d'identifier l'esprit supay responsable de l'ensorcèlement par une série d'interrogations successives. D'autre part, la multiplication des images sensibles décrivant son parcours dans l'espace offre simultanément la clé de la guérison obtenue. Cet usage rhétorique de l'interrogation et de la métonymie permet en effet au spécialiste de déplacer la focale des individus vers les espaces contigus dans lesquels se joue l'action rituelle. L'attention se détourne de la personne du chamane et de celle du patient, pour se focaliser sur la localisation de l'esprit responsable de la maladie, un espace instable et dépersonnalisé, celui de la maladie : ombre, vent, pluie, nuit, rivière, friche, liane... Un paysage associé à la maladie se dessine autour de l'évocation du nom. D'un point de vue formel, la structure paralléliste offre le support de cette quête, composée de noms de lieux variables - déclinés dans une forme syntaxique constante et particulièrement favorable à la mémorisation :

\begin{tabular}{|c|c|c|c|}
\hline Liste de noms & Suffixe locatif & $\begin{array}{l}\text { Prédicat } \\
+ \text { agentif }\end{array}$ & $\begin{array}{l}\text { Modalité : } \\
\text { question } \\
\text { rhétorique }\end{array}$ \\
\hline Ombre & /-punta/ & \multirow{7}{*}{$\begin{array}{l}\text { /puri-k/ } \\
\text { marcher, rôder- } \\
\text { AG } \\
\text { /tiya-k/ } \\
\text { être assis-AG } \\
\text { /shaya-k/ } \\
\text { se dresser-AG }\end{array}$} & \multirow{7}{*}{\begin{tabular}{|l} 
Supay-chu \\
Esprit-INTER \\
Est-ce l'esprit?
\end{tabular}} \\
\hline Vent & À l'extrémité, & & \\
\hline Pluie & frontière & & \\
\hline Nuit & /-uku/ & & \\
\hline Friche & À l'intérieur & & \\
\hline Arbre & & & \\
\hline Liane & & & \\
\hline
\end{tabular}

Hłbrildakqroa 7 (esprit-maître des singes) 


\begin{tabular}{|l|}
\hline /-washa/ \\
Derrière, de dos \\
\hline /-siki-pi/ \\
Au pied \\
\hline /-awa/ \\
Au-dessus
\end{tabular}

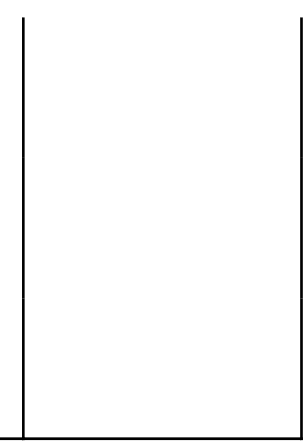

Dans les méandres de ce flot d'images, l'impossibilité d'énoncer ex abrupto le nom de l'esprit invoqué constitue la trame de la performance rituelle. Ce nom n'apparaît qu'en épilogue, au v. 15, avec la formule secrète «Aychakama» (inconnue des non-chamanes et réservée au langage rituel), directement adressée à l'interlocuteur invisible, se référant au maître des esprits-singes : celui qui chasse les humains, comme les humains chassent le gibier en forêt, en plantant un dard dans leur chair. La tension qui entoure la formulation du nom est aussi manifeste dans la performance d'un chamane du peuple Warao-Winikina de la vallée du delta de l'Orénoque au Vénézuela, étudiée par l'ethnomusicologue Dale Olsen. Ces paroles répétitives énoncées d'une voix monocorde par le chamane hoarotu se résument à la quête du nom d'un esprit-animal (hebu) responsable d'une maladie infantile ${ }^{24}$ :

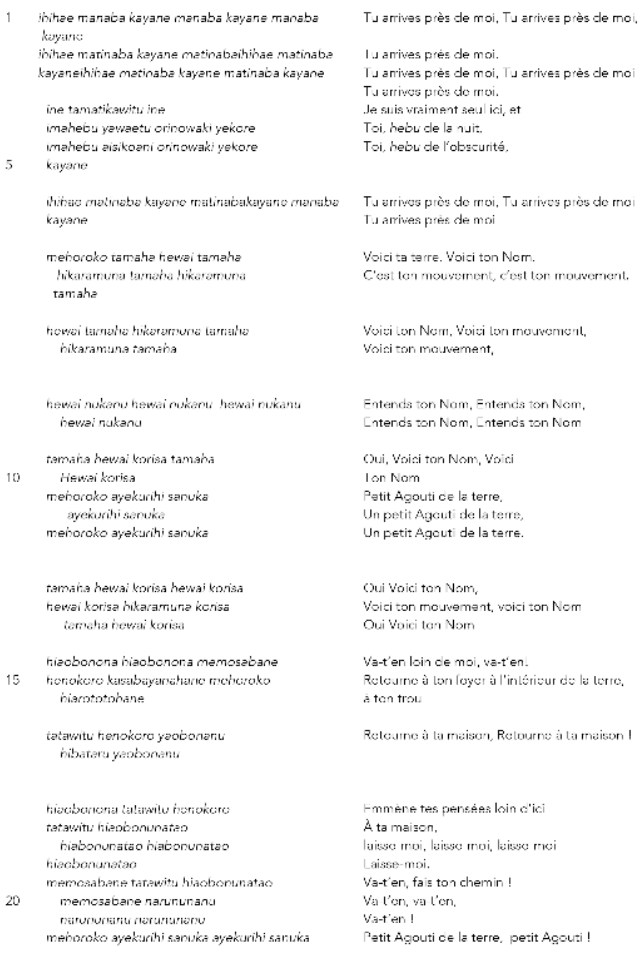

19 Alors que l'énonciation trace une carte mentale dans le chant précédent, elle en vient ici à épouser les contours et le "mouvement» des êtres qu'elle caractérise dans ce chant $d u$ " petit agouti de la terre ${ }^{25}$ ». Elle offre aussi à l'œil de l'imagination la seule trace « visible » d'un dialogue mené par le chamane, où se confond le patient et l'esprit destinataire dans une complicité de l'écoute : «Entends ton nom » figurant l'origine du mal hebu, pour se terminer sur une exhortation au départ : «Va-t'en loin de moi! [...] 
Emmène tes pensées loin d'ici [...] Laisse-moi !» (v. 16, v. 19, v. 22). Mais si l'évocation possède bien un début et une fin, clairement identifiables dans l'énonciation le renvoi -, et audible par défaut - le silence -, ce n'est que pour mieux cibler l'origine du malaise : la possibilité d'étendre à l'infini les descriptions de ces êtres pathogènes.

Dans le répertoire quechua, si l'énonciation du nom tend à paraître problématique, c'est une multiplicité de qualités sensibles qui lui est substituée comme l'unique voie de figuration possible de ces entités qui échappent aux principes de la relation de dénotation propre au langage ordinaire. Un chamane quechua décline ainsi une série d'images mentales, un kaléidoscope de couleurs revêtues par l'esprit d'un serpent responsable de la capture de l'âme d'un patient agité par la fièvre, conséquence d'une agression sorcellaire :

\begin{tabular}{|c|c|c|}
\hline \multirow[t]{4}{*}{1} & Uchpa uchpa tarunaka tarunay & Couleuvre grise comme la cendre! \\
\hline & Uchpa uchpa tarunay taruna taruna & Petite couleuvre grise comme la cendre \\
\hline & Imapitay wawaynipa animantaka? & Où donc se trouve le reflet de mon enfant? \\
\hline & apapashpa payka sirihushpan ukupishi & l'ayant emporté là où elle s'allonge, dit-on \\
\hline \multirow[t]{5}{*}{5} & apashkaylla apashkalla & I'ayant emporté avec elle, \\
\hline & Uchpa uchpa tarunay tarunay & Petite couleuvre grise! \\
\hline & Uchpa uchpa tarunay tarunay & Petite couleuvre grise! \\
\hline & Killu killu tarunayka taruna tarunay & Petite couleuvre verte! \\
\hline & Killu killu tarunay tarunay tarunay & Petite couleuvre verte! \\
\hline \multirow[t]{2}{*}{10} & $\begin{array}{l}\text { Inchichipi tarunayka tarunay tarunay } \\
\text { Killu killu tarunay tarunay } \\
\text { Maypi siririn chay ukupichu wawaynipa } \\
\text { animanta pitichishpa? } \\
\text { tarunay tarunay tarunay }\end{array}$ & $\begin{array}{l}\text { Coiffant la tête de l'esprit Inchichi, petite couleuvre, } \\
\text { petite couleuvre! } \\
\text { Petite couleuvre verte! } \\
\text { Dans l'espace creux où elle s'allonge, a-t-elle fait } \\
\text { couper le fil du reflet de mon enfant? } \\
\text { Petite couleuvre, petite couleuvre, petite couleuvre! }\end{array}$ \\
\hline & Killu killu tarunayka tarunay & Petite couleuvre verte! \\
\hline \multirow[t]{8}{*}{15} & Killu killu tarunay tarunay tarunay & Petite couleuvre verte! \\
\hline & Kaspi awapi siririk tarunayka tarunay & Au-dessus du tronc arraché, la petite couleuvre qui \\
\hline & Sirihu' ukupichu yaykuchishpa wawaynipa & s'allonge, \\
\hline & animantaka & Celle qui s'allonge, a-t-elle fait pénétrer à l'intérieur \\
\hline & Siririshpa tarunayka tarunay tarunay & le reflet de mon enfant? \\
\hline & & La petite couleuvre qui s'allonge, petite couleuvre! \\
\hline & Puka puka tarunay tarunay (...) & Petite couleuvre rouge de sang ! (...) \\
\hline & yana yana tarunayka tarunay tarunay & Petite couleuvre noire! \\
\hline 20 & Tarunay tarunay tarunay & Petite couleuvre petite couleuvre! \\
\hline
\end{tabular}

21 Plutôt qu'elle ne livre un diagnostic établi, une image aux contours nets et définis, ou un nom propre permettant de singulariser le référent du discours, la glose rituelle évoque sous un éventail chromatique - gris cendre, vert, rouge de sang, noir - des indices perceptifs ébauchant sous diverses facettes l'apparence visuelle des êtres invoqués. Ces évocations sont interrompues par des questions rhétoriques, la technique traditionnelle de l'erotesis. Ces questions portent sur le lieu de capture de l'«âmereflet », qui occupe du point de vue du chamane la position d'un « enfant ».

v. 3 Où donc se trouve le reflet de mon enfant?

v.12 Dans l'espace creux où elle s'allonge, a-t-elle fait couper le fil du reflet de mon enfant?

v.17 En s'allongeant, a-t-elle fait pénétrer à l'intérieur le reflet de mon enfant?

La déclinaison de ces couleurs s'inscrit dans un ordre syntaxique paralléliste évoquant un parcours, une quête diffractée dans l'énonciation, élément narratif commun à de nombreux répertoires quechua ou warao ${ }^{26}$. À moins de se résigner à l'idée qu'il n'y aurait au fond rien à transmettre, on peut examiner l'hypothèse que ces couleurs évoquées ne le sont pas accidentellement. Je suggère qu'elles caractérisent par défaut 
un mode de figuration possible des entités mises en présence. En effet, l'énoncé ne se réfère pas à un être singulier et unique ${ }^{27}$, pas plus qu'il ne viserait à transmettre une information selon les règles de la communication ordinaire : pertinence, économie des syntagmes, respect des conventions du dialogue... Ici, les qualités sensorielles sont évoquées à voix haute afin que le patient se livre lui-même à une forme d'introspection, à un acte de déchiffrement portant sur la forme visible des esprits qui l'ont ensorcelé. L'aspect lacunaire du signe, le caractère sibyllin du message transmis en offre le support. Certes, un tel exercice, hautement spéculatif, se détache d'une intuition première qui repose sur l'invisibilité matérielle, pour le patient, des êtres invoqués dans l'espace rituel. Néanmoins, cette ébauche de figuration chromatique se fonde sur un ensemble de savoirs implicites partagés portant sur le «monde possible » propre aux esprits invoqués, acquis par ouï-dire lors de l'apprentissage collectif des mythes. Les images énoncées tissent avec la trame du mythe un palimpseste. C'est notamment l'hypothèse que ces êtres que le chamane est seul à percevoir sont dotés d'ornements visibles, de coiffes colorées comme la robe du reptile, qu'ils peuvent apparaitre sous une forme anthropomorphe ou zoomorphe, que leurs qualités sensibles sont révélatrices de leur puissance, qu'ils sont capables de transformation, etc. C'est d'un tel soubassement d'images mentales que le chant est la traduction ${ }^{28}$.

La force évocatrice propre aux paroles rituelles se voit en outre renforcée par des dispositifs matériels et techniques. En effet, la musicalité de la voix ondulant d'une octave à celle en dessous à chaque répétition du même vers, s'accompagne de gestes éminemment suggestifs. De la poudre de roucou, une plante tinctoriale amazonienne de couleur vermillon comme la "petite couleuvre de sang" responsable de l'ensorcèlement, est étalée sur le front du patient, tandis que la guérisseuse caresse le corps du malade à l'aide d'une fleur de coton jaune afin d'en extraire ou "balayer " (pichana) la douleur et la fièvre, symptômes qui ne sont quasiment jamais nommés frontalement dans les chants ikara. En d'autres termes, avec une économie de moyens linguistiques et gestuels extraordinaire, le chamane parvient à suggérer plutôt qu'à dire explicitement l'identité de nouveaux acteurs sur la scène rituelle. L'essentiel du message cependant ne réside pas dans la définition d'une identité. C'est plutôt l'indétermination qui est en jeu, en des termes qu'en philosophie du langage Quine nommerait l'inscrutabilité de la référence ${ }^{29}$. Ces chants ikara ne veulent en un sens rien dire. Ou plus exactement, ce qu'ils disent n'est pas qu'il n'y a rien, mais que rien ne peut être énoncé que sous une forme et un dispositif qui échappent à toute communication fondée sur la transparence du sens.

\section{Variations et voix enchâssées}

Revenons sur le champ à la situation thérapeutique elle-même. La scène du rituel chez les peuples Runa d'Amazonie péruvienne n'a rien de spectaculaire. Ce qui s'y joue est une adhésion lente et imperceptible de chacun à une pièce dont il est un acteur avec les autres, sans jamais pour autant saisir la signification totale de l'événement ayant cours ici et maintenant. C'est dans ce climat incertain mais hautement suggestif que se constitue le terreau fécond de l'adhésion au rituel. Mon ami Yana Shunku, chamane runa d'Amazonie péruvienne, est accroupi au dessus d'un malade étendu sur la claie en écorce de palmier qui sert de plancher. Ce soir, il officie dans la plus parfaite obscurité, palpe le corps à plusieurs endroits : il s'arrête sur la mollesse du cou, caresse le front 
délicatement, soupèse les mains, palpe le ventre, le foie. Il cherche dans les mains du malade une réponse : sueur, chaleur ou frisson, palpitations... Le pouls est un indice infaillible, il me dit l'entendre comme une voix qui tressaille et exprime la douleur par des sons que lui-seul peut entendre «tsiik tsiik tsiik». Lui et son patient. Car c'est aussi le principal symptôme invoqué par le malade à son arrivée, lorsqu'il décrit ses maux au spécialiste. Le malade est un vieil homme d'une cinquantaine d'années, excellent chasseur, qui aurait été victime d'une rencontre d'esprit à la lagune située à quelques heures de marche du village. Il chuchote ces paroles en arrivant :

Umaynika muyurin, lismayashpa purini, yanah'lla rikuni, tsik tsiik ruran

[Ma tête tourne, j'ai des vertiges en marchant, je vois tout noir, cela fait tsiik tsiik

(dépliant son bras et caressant son pouls).]

Le chamane ne semble guère y prêter plus d'attention. Il semble ailleurs, il manipule son cigare en le faisant rouler dans la paume de ses mains et souffle dessus légèrement. Puis il se retourne vers le fils du malade accroupi à quelques mètres. Muskushkankichu ? ("As-tu rêvé?»). Son interlocuteur Nemesio reste de marbre. Quelques minutes passent. Un observateur extérieur n'entendra que le craquement du plancher d'écorce sous le poids des corps accroupis ou étendus. Le chamane continue de palper le corps souffrant. Nemesio se confie enfin :

Kayna washa sacha chawpimanta shamushpayni... Yana sumbriruyu atun ruku umayushi chasnallatata... Imasnaram?

[Il y a quelques jours lorsque je suis rentré des terres du milieu de la forêt... Avec un chapeau noir, il a une énorme tête, dit-on, c'est cela-même... Qu'est-ce que cela peut bien être ?]

Le chamane écoute attentivement, puis reste muet à son tour. Le dialogue n'est guère plus étoffé. Assis en tailleur, Yana Shunku chuchote des paroles inaudibles, la paume de la main recourbée en forme de tube sur son cigare, placée juste devant sa bouche. Ses murmures sont interrompus par de brèves expirations, suivies d'une expiration plus lente, qui semble terminer une longue phrase incompréhensible et difficile à prononcer. L'élocution est extrêmement rapide et monotone. Plus tard, il confirme que l'esprit de la raie noire (Raya Supay) est celui qui a fléché un dard invisible dans le corps du chasseur affaibli par une longue marche sur le chemin du retour au village. Les symptômes qu'offre le malade sont l'égarement, les vertiges, la fièvre chronique (une sensation de chaleur oppressante dans la poitrine), un sentiment vague de tristesse et d'abattement. Quels sont les mots employés pour dire de tels maux? C'est d'une tristesse singulière dont se plaint le malade lorsqu'il affirme qu'il se meurt littéralement :

Ayaaw wawayni, llakipata wañuhunimi

[Aaah mon fils... je meurs vraiment de mélancolie.]

Le chamane s'engage dans un combat. Il va lancer ses propres "flèches ${ }^{30}$ » (supay wiruti), terme consacré pour désigner les esprits auxiliaires à l'assaut des esprits responsables de l'ensorcèlement. Mais si l'on s'en tient à ce qui est proprement énoncé, à ce qui est entendu et vu, un observateur extérieur - l'ethnographe et le patient profane partagent une condition analogue - est forcé de constater que les indices de la présence de ces entités sont extrêmement ténus, pour ne pas dire nuls. Le premier temps est ici celui de l'appel.

$28 \mathrm{Au}$ moment d'entamer la cure, le yachak se livre à une captatio benevolentiae: il convoque ses esprits auxiliaires par une adresse vocative directe en les nommant ses propres "aïeux », établissant ainsi un lien de filiation directe avec ces derniers, avec 
lesquels il partage en quelque sorte une même chair. Au même moment, il souffle lentement la fumée de son cigare et avale quelques gorgées d'une eau de vie contenue dans une calebasse, avant d'entonner l'incantation dont voici la transcription :

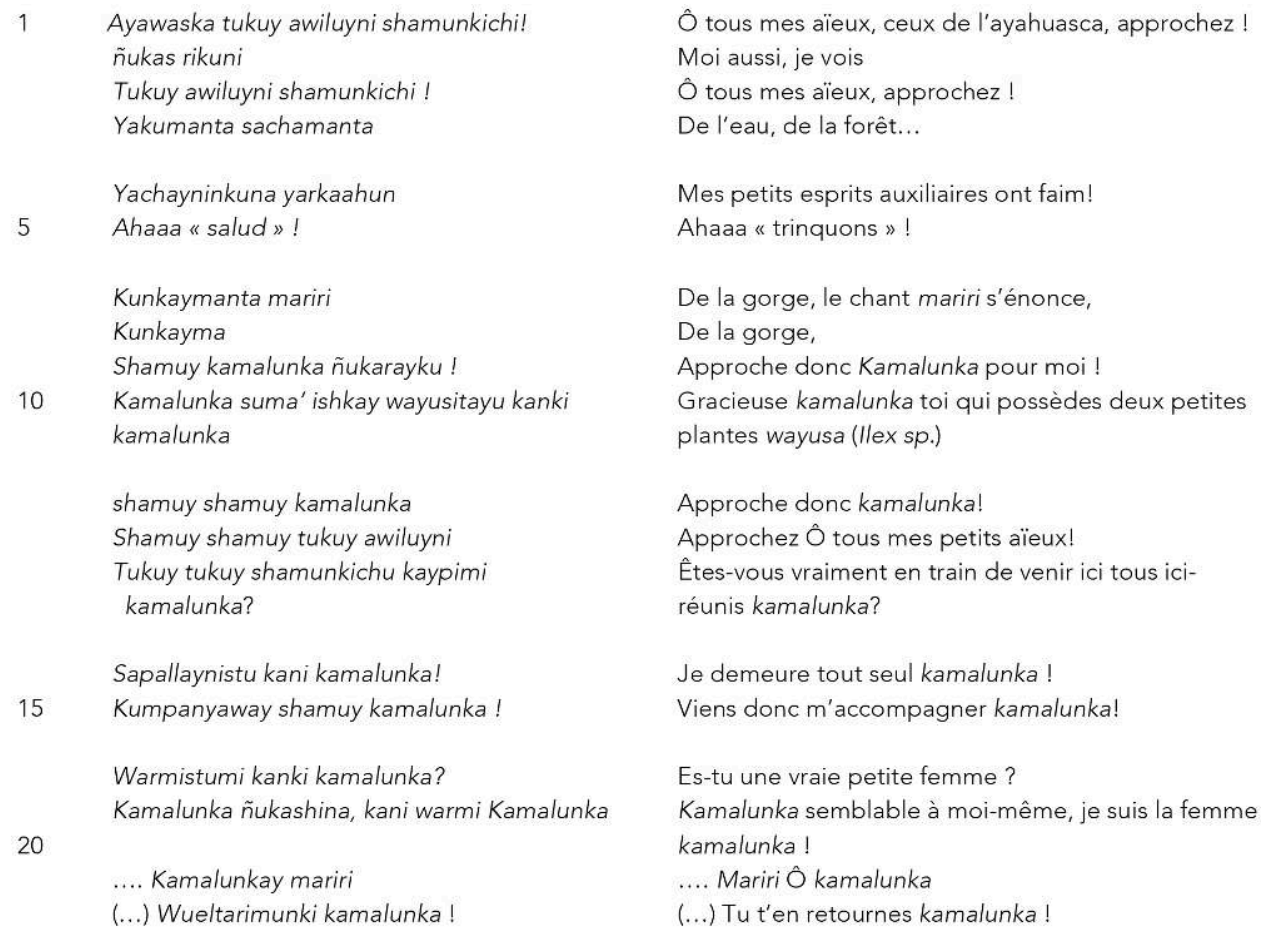

Une cohorte d'esprits entre en scène: ceux des plantes ingérées lors de l'initiation chamanique, le fameux breuvage psychotrope ayahuasca ${ }^{31}$ ou la graine de Strychnos sp. kamalunka, ingérée lors de son apprentissage. Ces esprits auxiliaires sont réputés se loger dans une substance flegmatique appelée communément mariri, soit un «chant, flegme » censé être énoncé ou expectoré au moment de la cure. Le chamane nomme ses esprits « aïeux » (awilu), ou «mes esprits» (supayninkuna), le terme supay dénotant de façon ambivalente démons et entités auxiliaires. La "mythologie implicite " des chamanes, celle qui émane des récits d'initiation, identifie ces esprits à des dards contenus dans leur propre corps. Ce corps est considéré comme analogue à un tube à projectile - littéralement une sarbacane (pukuna) ${ }^{32}$. Toutefois l'entrée en scène du chamane est celle d'un être affamé et assoiffé, à l'image de ces êtres qu'il convoque et qu'il s'empresse de nourrir avec du tabac et de l'alcool. La frontière entre les corps se voit ainsi partiellement abolie au cours du rituel. En effet, au moment où le chamane s'abreuve d'une boisson hautement alcoolisée, ce sont les esprits qui se nourrissent à travers son propre corps. Ce processus de condensation du métabolisme rituel en la personne du spécialiste retient l'attention. Car c'est au fond la condition même de l'adhésion possible des acteurs du rituel à la cure. C'est au terme de l'initiation qui consiste à ingérer des substances pour la plupart toxiques et douloureuses, que le chamane en vient à avaler et contenir dans son propre corps des corps étrangers : larves lumineuses (kuru), fléchettes (supay wiruti), éléments minéraux, le cas échéant. L'initiation donne lieu à un processus de subjectivation complexe au cours duquel le corps de l'apprenti chamane douloureusement affaibli par la réclusion, parfois l'ingestion de psychotropes, devient le réceptacle de nouvelles entités, d'acteurs, dont son corps transformé porte la trace, et "re-présente » comme icône et index - indice d'une "présence » - à la fois. Lorsque le spécialiste rituel se nourrit, lorsqu'il boit au 
cours de la performance rituelle, l'action peut être considérée tout à la fois comme réflexive - il se nourrit - et transitive - il nourrit ses esprits, ce qu'atteste la joyeuse exhortation à l'ébriété «Ahaaa trinquons!» (v.6). Le principe de l'adhésion au rituel chamanique repose en partie sur l'acceptation de cette clause. La formule autoréférentielle "Kamalunka semblable à moi-même, je suis la femme kamalunka!» (v. 18 Kamalunka ñukashina kani Kamalunka), fait de la voix du chamane l'enchâssement d'une voix quasi autre, elle-même également frappée par le principe d'indétermination. Il est en fait remarquable que lorsqu'il profère ces paroles, son expérience d'initié (pour qui des êtres dont l'apparence est informulable sont devenues "visibles ») et celle de thérapeute humain (être de chair présent ici et maintenant, devant le patient) viennent se superposer ou se condenser pour dispenser de son être une image tout à fait indiscernable. Le modalisateur comparatif -shina (" pareil à »), qui entérine le doute et rend possible à la fois la croyance, tisse le lien entre le locuteur actuel et cette "petite femme " végétale nommée kamalunka, membre d'une troupe d'esprits auxiliaires sommés de prendre place dans le rituel à travers la voix du récitant.

Le contexte de l'action rituelle se duplique : c'est à la fois le chamane qui énonce son chant et les esprits qui sortent littéralement de sa propre bouche. Cette réflexivité de l'action rituelle se met en place à la faveur de la construction d'un nouveau champ déictique qui constitue le "méta-contexte» de l'action rituelle : shamunkichu kaypimi ("venez-vous ici-même?»), le déictique proximal ici couplé à un suffixe évidentiel insistant sur la vérité ou l'ostension du référent - mi, laissant supposer que cet espace est « ici », « devant lui », ancré dans l'expérience actuelle. Ce qui importe n'est pas tant le transfert d'informations, le principe de non contradiction ou l'économie de la parole, mais la mise en place de ce nouvel espace d'interaction entre les participants. Après avoir énoncé à voix haute ces paroles, le chamane les transpose dans son souffle musical qui épouse les contours mélodiques du chant. C'est le recours à l'expressivité corporelle qui prime, la concentration, le recueillement, la musicalité de la voix, le sifflement extrêmement codifié de la fumée expectorée sur le corps souffrant, qui se situe en deçà et au-delà du champ discursif. Le yachak peut clôturer son dialogue par un protocole de départ semblable aux salutations ordinaires: (v. 19) "va-t'en Kamalunka!».

Que se passe-t-il du point de vue du patient? La douleur qu'il éprouve vient trouver une expression singulière dans le champ des affects, à travers la dimension à la fois descriptive et prescriptive qu'ils revêtent dans la performance. En atteste ce chant de la démence destiné à guérir un chasseur égaré33: 


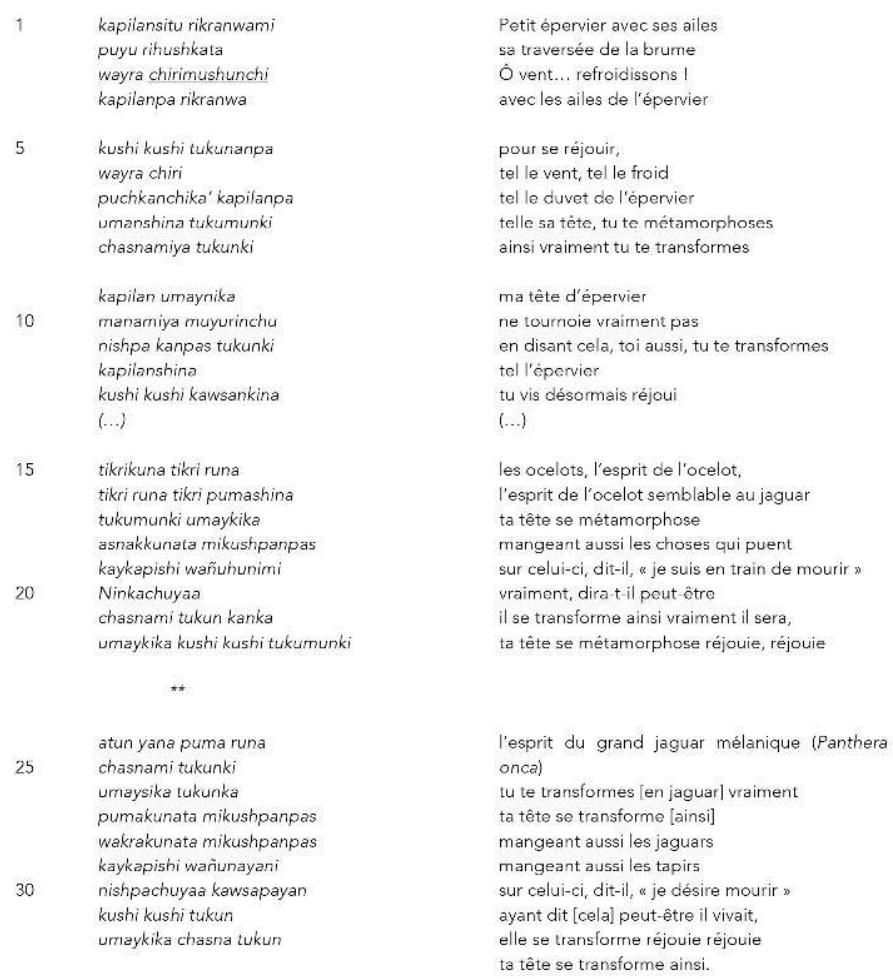

Le glissement des embrayeurs personnels (pronoms de la première, deuxième et troisième personne) offre l'exemple d'un dialogue singulier, où la permutation possible des destinataires rend la scène inextricable. L'énonciateur occupe en effet une position multiple. Il adopte la figure de l'épervier, de l'ocelot ou du jaguar puis prête son corps et sa voix au patient pour dire la guérison, l'apaisement de la fièvre, dans un va-et-vient incessant entre la première et la seconde personne : «ma tête d'épervier ne tournoie vraiment pas » (v. 10-11), «ta tête se métamorphose réjouie» (v. 22). Un véritable concert de voix enchâssées est manifeste dans ce chant de la démence, où les affects contradictoires du patient (joie, v. 19 ; désir de mort, v. 28) sont évoqués comme la face et l'envers d'une même relation, œuvrant ici à la façon d'un interrupteur. La distinction logique opposant des émotions comme la peine et la réjouissance viendrait se substituer à la distinction muette qui oppose souffrance et guérison dans l'expérience du malade. Cette distinction fait écho à l'expression intime des maux par le vieux patient pris de vertige, qui ressentait tout à l'heure au plus profond de son être cet obscur « désir de mort » (wañu-naya-) faisant irruption dans la maladie.

En somme, plutôt qu'il ne viendra clore l'énigme posée par Lévi-Strauss à la découverte du chant de l'indien Cuna Guillermo Haya, ce parcours a l'ambition plus modeste d'ouvrir des voies qu'une anthropologie pragmatique peut explorer, à condition de renoncer à un paradigme de la communication fondé sur le déchiffrement d'une intention signifiante, le principe de pertinence et la quête de la dénotation. J'ai ébauché le portrait d'une performance où les mots, les maux et les images-objets rituels constituent des traductions possibles de «mondes possibles». Ce que le chamane runa d'Amazonie rend visible est l'impossibilité qu'il éprouve à figurer explicitement les entités présentes sur la scène rituelle et le paradoxe de l'acte de nomination qui "multiplie» plutôt qu'il ne parviendrait à singulariser un être ou un acteur. La présence d'une multiplicité d'acteurs peut en effet être inférée des images mentales déclinées dans ces chants. La particularité des images rituelles tient à leur condition de 
fabrication in situ, qu'elles soient énoncées ou figurées par d'autres modalités sémiotiques, geste, souffle, fumée de tabac, et à leur statut temporel. Ces images ne pourront jamais totalement s'affranchir des actes de fabrication ou de perception par lesquels elles ont vu le jour. La performance chamanique offre en effet un contexte où chaque image indexe et affecte avant tout une relation, plutôt que des termes pris isolément. C'est précisément la contagion des images, le fait de partager une même vision - avec toutes les difficultés que cela peut impliquer - qui définira la position des acteurs, leur identité ou leur "face » pour emprunter la terminologie goffmanienne. Parmi les Runa, c'est ainsi le fait d'observer qu'une raie sert de coiffe élégante, un poisson-silure de ceinture et un anaconda de hamac, chez les esprits de l'eau Tsunki, qui définit la position du chamane yachak comme analogue à celle de ses congénères non humains. Mais l'analogie rencontre ses propres limites. La complicité décrite par les initiés à travers le partage d'un acte de vision est une complicité d'ordre figuratif : elle émerge ici du partage des conditions de la figuration elles-mêmes, dont les caractéristiques évoquées dans ce texte sont l'indétermination de la référence, la répétition et l'inachèvement. Les variations de l'image rituelle - la voix chantée, "soufflée " avec la fumée de tabac, ou sifflée - sont autant de techniques visant à soumettre le monologue du chamane à la forme dialogique, l'image à sa dimension temporelle, inachevée.

\section{BIBLIOGRAPHIE}

BAUMAN Richard et SHERZER Joel (dir.), Explorations in the Ethnography of Speaking [1974], Cambridge, Cambridge University Press, 1989.

BLoch Maurice, Essays on Cultural Transmission, Oxford, Berg Publishers, 2005.

CESARINO Pedro N., « Entre la parole et l'image. Le système mythopoétique Marubo », Journal de la Société des américanistes, vol. 97, n 1, 2011, p. 223-257.

DÉLÉAGE Pierre, Le Chant de l'anaconda. Enquête sur l'épistémologie rituelle du chamanisme sharanahua, Nanterre, Société d'ethnologie, 2009.

FONTAINE Laurent, « Les cours d'eaux dans les incantations chamaniques des indiens Yucuna (Amazonie colombienne) », Journal de la Société des américanistes, vol. 97, n 1, 2011, p. 119-150.

Fox James J., « « Our Ancestors Spoke in Pairs » : Rotinese View of Language, Dialect, and Code », in Richard BAUMAN et Joel SHERZER (dir.), Explorations in the Ethnography of Speaking [1974],

Cambridge, Cambridge University Press, 1989, p. 65-85.

GOODMAN Nelson, Ways of Worldmaking, Brighton, Harvester Press, 1978.

GRICE H. Paul, « Meaning », Philosophical Review, n67, 1957, p. 377-388.

GUTIERREZ CHOQUEVILCA Andrea-Luz, "Sisyawaytii tarawaytii. Sifflements serpentins et autres voix d'esprits dans le chamanisme quechua du haut Pastaza (Amazonie péruvienne) », Journal de la Société des américanistes, vol. 97, n 1, 2011, p. 179-222. 
HANKS William, « Comment établir un terrain d'entente dans un rituel ? », dans SEVERI Carlo et BONHOMmE Julien (dir.), Paroles en actes, Cahiers d'anthropologie sociale, vol. 5, Paris, L'Herne, 2009, p. 87-115.

JAKOBSON Roman, « On Linguistic Aspects of Translation », On Translation 3, 1959, p. 232-39.

KOPENAWA Davi et AlBert Bruce, La Chute du ciel. Paroles d'un chaman Yanomami, Paris, Plon, 2010.

KRIPKE Saul, Naming and Necessity, Oxford, Blackwell, 1972.

LÉVI-STRAUSS Claude, Anthropologie structurale, Paris, Plon, 1958.

LÉVI-STRAUSS Claude, L’Homme nu, Paris, Plon, 1971.

LUNA Luis Eduardo, «Icaros : Magic Melodies Among the Mestizo Shamans of the Peruvian Amazon ", in E. Jean Matteson LANGDON et Gerhard BAER (dir.), Portals of Power : Shamanism in South America, Albuquerque, University of New Mexico Press, 1992, p. 231-253.

MONOD-BECQUELIN Aurore, « Le sang et le corps, ou le blanc et le noir ? Contribution à l'étude du parallélisme dans la tradition orale maya », Journal de la Société des américanistes, n 72, Paris, 1986, p. 7-31.

MONOD-BECQUELIN Aurore, « Le tour du monde en quelques couplets. Le parallélisme dans la tradition orale maya », in Jocelyne FERNÁNDEZ-VEST (dir.), Kalevala et traditions orales du monde, Paris, Éditions du CNRS, 1987, p. 467-488.

MONOD-Becquelin Aurore, « Monologues polyphoniques. Les discours rituels maya », in Nicole REVEL et Diana REY-HULMAN (dir.), Pour une anthropologie des voix, Paris, L'Harmattan/INALCO, 1993, p. $295-327$.

OVERING Joana « The Shaman as a Maker of Worlds », Man, New Series, vol. 25, n 4, 1990, p. 602-619.

OLSEN Dale, « Magical Protection Songs of the Warao Indians », Latin American Music Review, vol. 1, $\mathrm{n}^{\circ} 2,1980, \mathrm{p} .131-161$

OLSEN Dale, Music of the Warao of Venezuela: Song People of the Rain Forest, Gainesville, University Press of Florida, 1996.

QUINE Willard Van Orman, Word and Object, Cambridge [MA], MIT Press, 1960.

SHERZER Joel, Kuna Ways of Speaking. An Ethnographic Perspective [1983], Tucson, Hats off Books, 2001.

SEEGER Anthony, Why Suyá Sing? A Musical Anthropology of an Amazonian People, Cambridge, Cambridge University Press, 1987.

SEVERI Carlo, Le Principe de la chimère. Une anthropologie de la mémoire, Paris, Aesthetica/Éditions Rue d'Ulm/Musée de Quai Branly, 2007.

SEVERI Carlo et BONHOMME Julien (dir.), Paroles en actes, Cahiers d'anthropologie sociale, vol. 5, Paris, L'Herne, 2009.

SEVERI Carlo, « Transmutating Beings : A Proposal for an Anthropology of Thought », Hau, vol. 4, $n^{\circ} 2,2014$.

SHERZER Joel, Verbal Art in San Blas. Kuna Culture through its Discourse, Cambridge, Cambridge University Press, 1990. 
SHERZER Joel, Kuna Ways of Speaking. An Ethnographic Perspective [1983], Tucson, Hats off Books, 2001.

SPERBER Dan, La Contagion des idées : théorie naturaliste de la culture, Paris, Odile Jacob, 1996.

SPERBER Dan et WILSON Deirdre, La Pertinence : communication et cognition, Paris, Minuit, 1989.

TAYLOR Gérald, « Supay », Amerindia, n 5, 1980, p. 47-63.

TOWNSLEY Graham, « Song Paths : the Ways and Means of Yaminahua Shamanic Knowledge », L'Homme, n 126-128, vol. 33, 1993, p. 449-468.

\section{NOTES}

1. Les Runa attribuent aux syntagmes supay, aya et alma une signification qui varie contextuellement. Ces termes peuvent être traduits par "démon» (supay), "spectre, fantôme » (aya) et "reflet, âme" (alma)]. Leur polysémie émane d'une opération de resémantisation prenant place pendant la période coloniale, à l'arrivée des premiers missionnaires espagnols (voir Gérald Taylor, «Supay », Amerindia, n 5, 1980, p. 47-63). Ces " esprits » apparaissent et disparaissent aussi vite qu'ils sont apparus et correspondent davantage à des images mentales contextuellement associées à une faculté d'affecter, d'agir sur. Les conditions de vision et de perception de ces images sont soumises à des contraintes souvent exclusives (jeûne drastique, abstinence sexuelle, état cataleptique, narcose, solitude). Au point qu'elles justifieraient la qualification par défaut, frappée de négativité dans la terminologie familière aux anthropologues, d'«in-visible» pour « esprit». Ces deux notions, saisies hors contexte ethnographique, ne signifieraient au fond rien d'autre qu'un déficit de visibilité de l'objet dénoté dans des conditions perceptives ordinaires, et il n'est guère productif de les tenir pour acquises ou dotées d'une signification concrète isolée de leur contexte ethnographique, mais surtout de leur contexte de transmission.

2. Il est aujourd'hui impensable de considérer que la pensée religieuse des sociétés traditionnelles se réduit à des processus narratifs isolés de tout contexte historique. D'importantes contributions récentes ont montré que la mémoire sociale et culturelle se déploie autant à travers le discours explicite qu'à travers des rites et des actions non verbales dont la performance garantit la persistance et la transmission parmi un collectif ou un ensemble culturel donné (Dan Sperber, La Contagion des idées : théorie naturaliste de la culture, Paris, Odile Jacob, 1996 ; Maurice Bloch, Essays on Cultural Transmission, Oxford, Berg Publishers, 2005). Une application féconde de cette approche à des matériaux américanistes est explorée par Pierre Déléage, Le Chant de l'anaconda. Enquête sur l'épistémologie rituelle du chamanisme sharanahua, Nanterre, Société d'ethnologie, 2009 ; Carlo Severi et Julien Bonhomme (dir.), «Paroles en actes ", Paris, L'Herne, «Cahiers d'anthropologie sociale », vol. 5, 2009 ; Pedro N. Cesarino, « Entre la parole et l'image. Le système mythopoétique Marubo ", Journal de la Société des américanistes, vol. 97, nº 1 , 2011).

3. Nelson Goodman, Ways of Worldmaking, Brighton, Harvester Press, 1978.

4. Une comparaison terme à terme de son approche de l'art avec l'ethnographie des performances chamaniques a été esquissée par Overing à partir de matériaux piaroa (Joana Overing, "The Shaman as a Maker of Worlds ", Man, New Series, vol. 25, n 4, 1990). Cet article ne vise pas à se prêter à un tel exercice.

5. Ces sociétés quechuaphones de chasseurs-cueilleurs-essarteurs - environ 4200 individus en 2014 - occupent la vallée alluviale du fleuve Pastaza, un affluent septentrional de l'Amazone au Pérou. Les Runa (autodénomination ethnique dénotant l'« humain », l'« esprit », les " gens ») sont intermariés avec les membres de l'ensemble Jivaro et Zaparo-Andoa. Les circonstances de leur 
ethnogenèse coloniale et leur formation pluriethnique sont pas sans lien avec le fait qu'ils excellent dans l'art de la traduction interlinguistique et maitrisent d'autres formes de communication iconique (Kohn, 2002 ; Andrea-Luz Gutierrez Choquevilca, « Sisyawaytii tarawaytii. Sifflements serpentins et autres voix d'esprits dans le chamanisme quechua du haut Pastaza [Amazonie péruvienne] », Journal de la Société des américanistes, vol. 97, nº 1, 2011).

6. Claude Lévi-Strauss, "L'efficacité symbolique", Anthropologie structurale, Paris, Plon, 1958, p. 13-14.

7. «À des images de la femme gisante dans son hamac ou dans la position obstétricale indigène, genoux écartés et tournée vers l'est, gémissante, perdant son sang, la vulve dilatée et mouvante [...] succèdent les appels nominaux aux esprits : ceux des boissons alcooliques, ceux du vent, des eaux et des bois, et même - témoignage précieux de la plasticité du mythe - celui « du paquebot argenté de l'homme blanc» (Claude Lévi-Strauss, Anthropologie structurale, Paris, Plon, 1958, p. 213-14).

8. Claude Lévi-Strauss, Anthropologie structurale, Paris, Plon, 1958, p. 209.

9. Claude Lévi-Strauss, Anthropologie structurale, Paris, Plon, 1958, p. 215-216.

10. L'anthropologie place en effet cet enjeu de la construction d'un «terrain d'entente » au centre du débat sur la question rituelle (William Hanks, «Comment établir un terrain d'entente dans un rituel ?», in Carlo Severi et Julien Bonhomme (dir.), Paroles en actes, Paris, L'Herne, «Cahiers d'anthropologie sociale », vol. 5, 2009.

11. Claude Lévi-Strauss, Anthropologie structurale, Paris, Plon, 1958, p. 218.

12. Voir par exemple Carlo Severi, «Transmutating Beings : A Proposal for an Anthropology of Thought ", Hau, vol. 4, n² 2, 2014.

13. Citons l'opération structurale de mise en forme des affects et de la pensée, que Claude LéviStrauss situe au fondement de l'efficacité symbolique et qu'il considère commune à la psychanalyse: "Il s'agirait chaque fois d'induire une transformation organique, consistant essentiellement en une réorganisation structurale, en amenant le malade à vivre intensément un mythe, tantôt reçu, tantôt produit, et dont la structure serait, à l'étage du psychisme inconscient, analogue à celle dont on voudrait déterminer la formation à l'étage du corps. L'efficacité symbolique consisterait précisément dans cette "propriété inductrice » que posséderaient, les unes par rapport aux autres, des structures formellement homologues pouvant s'édifier, avec des matériaux différents, aux différents étages du vivant : processus organiques, psychisme inconscient, pensée réfléchie " (Claude Lévi-Strauss, Anthropologie structurale, Paris, Plon, 1958, p. 222-223).

14. Joel Sherzer, Verbal Art in San Blas. Kuna Culture through its Discourse, Cambridge, Cambridge University Press, 1990 ; Id., Kuna Ways of Speaking. An Ethnographic Perspective [1983], Tucson, Hats off Books, 2001; Fox, James J., " "Our Ancestors Spoke in Pairs" : Rotinese View of Language, Dialect, and Code ", dans Richard Bauman et Joël Sherzer (dir.), Explorations in the Ethnography of Speaking [1974], Cambridge, Cambridge University Press, 1989; Aurore Monod-Becquelin, «Le sang et le corps, ou le blanc et le noir ? Contribution à l'étude du parallélisme dans la tradition orale maya ", Journal de la Société des américanistes, vol. 72, Paris, 1986 ; Ead., « Le tour du monde en quelques couplets. Le parallélisme dans la tradition orale maya », dans Jocelyne FernándezVest (dir.), Kalevala et traditions orales du monde, Paris, Éditions du CNRS, 1987 ; Ead., "Monologues polyphoniques. Les discours rituels maya", dans Nicole Revel et Diana ReyHulman (dir.), Pour une anthropologie des voix, Paris, L'Harmattan/INALCO, 1993; Joana Overing, "The Shaman as a Maker of Worlds", Man, New Series, vol. 25, n 4, 1990 ; Carlo Severi, Le Principe de la chimère. Une anthropologie de la mémoire, Paris, Aesthetica/Éditions Rue d'Ulm/ Musée de Quai Branly, 2007 ; Pierre Déléage, Le Chant de l'anaconda. Enquête sur l'épistémologie rituelle du chamanisme sharanahua, Nanterre, Société d'ethnologie, 2009 ; Andrea-Luz Gutierrez Choquevilca, "Sisyawaytii tarawaytii. Sifflements serpentins et autres voix d'esprits dans le 
chamanisme quechua du haut Pastaza (Amazonie péruvienne)», Journal de la Société des américanistes, vol. 97, $\mathrm{n}^{\circ} 1,2011$.

15. Roman Jakobson, « On linguistic Aspects of Translation », On Translation 3, 1959, p. 233.

16. Claude Lévi-Strauss, L'Homme nu, Paris, Plon, 1971, p. 600-601.

17. On renverra le lecteur à l'analyse consacrée par Saul Kripke à cette question en dehors de tout contexte rituel (Saul Kripke, Naming and Necessity, Oxford, Blackwell, 1972).

18. Andrea-Luz Gutierrez Choquevilca, «Sisyawaytii tarawaytii. Sifflements serpentins et autres voix d'esprits dans le chamanisme quechua du haut Pastaza (Amazonie péruvienne) », Journal de la Société des américanistes, vol. 97, n 1, 2011.

19. Andrea-Luz Gutierrez Choquevilca, «Sisyawaytii tarawaytii. Sifflements serpentins et autres voix d'esprits dans le chamanisme quechua du haut Pastaza (Amazonie péruvienne) », Journal de la Société des américanistes, vol. 97, n 1, 2011.

20. Graham Townsley, «Song Paths : the Ways and Means of Yaminahua Shamanic Knowledge », L'Homme, n० 126-128, vol. 33, 1993, p. 465.

21. L'ensemble de ces maximes permet, entre autres, de résoudre des questions liées à la sousdétermination linguistique des énoncés. Lorsqu'une ou plusieurs maximes sont violées, l'interlocuteur est amené à formuler des hypothèses et des interprétations au-delà de ce que dit explicitement l'énoncé, en remontant à l'intention communicative du locuteur. En effet, un grand nombre d'informations ne sont pas explicites et ne peuvent être déduites du sens logique des énoncés. Ces hypothèses, aussi nommées «implicatures» dans l'analyse pragmatique gricéenne, assimilent la communication humaine à un processus inférentiel de déchiffrement plutôt qu'à un modèle du code (Dan Sperber et Deirdre Wilson, La Pertinence: communication et cognition, Paris, Minuit, 1989).

22. Anthony Seeger, Why Suyá Sing? A Musical Anthropology of an Amazonian People, Cambridge, Cambridge University Press, 1987 ; Dale Olsen, Music of the Warao of Venezuela: Song People of the Rain Forest, Gainesville, University Press of Florida, 1996; Joana Overing, "The Shaman as a Maker of Worlds ", Man, New Series, vol. 25, n 4, 1990 ; Pierre Déléage, Le Chant de l'anaconda. Enquête sur l'épistémologie rituelle du chamanisme sharanahua, Nanterre, Société d'ethnologie, 2009 ; Pedro N. Cesarino, «Entre la parole et l'image. Le système mythopoétique Marubo », Journal de la Société des américanistes, vol. 97, n 1, 2011.

23. Cette technique est massivement répandue, notamment dans les chants rituels yucuna ou marubo (Laurent Fontaine, "Les cours d'eaux dans les incantations chamaniques des indiens Yucuna [Amazonie colombienne] ", Journal de la Société des américanistes, vol. 97, n 1, 2011 ; Pedro N. Cesarino, « Entre la parole et l'image. Le système mythopoétique Marubo », Journal de la Société des américanistes, vol. 97, $\mathrm{n}^{\circ}$ 1, 2011, p. 223-257).

24. Dale Olsen, "Magical Protection Songs of the Warao Indians ", Latin American Music Review, vol. $1, \mathrm{n}^{\circ} 2,1980$, p. 135-136.

25. La même stratégie de dénomination oblique prévaut ici comme dans tous les autres répertoires: le terme rituel consacré à l'entité invoquée pour le "petit agouti de la terre » (ayekurihi) diverge de celui employé dans la langue warao ordinaire : kahamuru en winikina ou kuhuamare en wayo pour l'espèce Dasyprocta aguti (Dale Olsen, « Magical Protection Songs of the Warao Indians", Latin American Music Review, vol.1, n², 1980, p. 136). L'ethnomusicologue recueillait plus d'une vingtaine de chants dédiés à divers esprits animaux hebu (iguane, tortue, anaconda, tapir, jaguar, daguet, opossum, fourmilier, etc.) dont la composition est strictement analogue sur le plan grammatical et narratif : description du lieu, du mouvement, du nom, puis renvoi des entités.

26. Voir Andrea-Luz Gutierrez Choquevilca, "Sisyawaytii tarawaytii. Sifflements serpentins et autres voix d'esprits dans le chamanisme quechua du haut Pastaza (Amazonie péruvienne)", Journal de la Société des américanistes, vol. 97, n 1, 2011 ; Dale Olsen, « Magical Protection Songs of 
the Warao Indians ", Latin American Music Review, vol. 1, n², 1980, et Id., Music of the Warao of Venezuela : Song People of the Rain Forest, Gainesville, University Press of Florida, 1996.

27. Seul un nom propre s'immisce dans cette évocation presque exclusivement sensible, celui d'Inchichi, maître du gibier connu dans la mythologie runa, car l'esprit du serpent en est l'auxiliaire familier.

28. Un témoignage autobiographique comme celui de Davi Kopenawa, chamane et leader Yanomami accédant à la vision des esprits de la forêt xapiri, conforte cette hypothèse : les xapiri sont assimilés à des réfractions d'images à l'infini : les miroirs, la luminosité et l'ornementation des xapiri sont le plus souvent invoqués (Davi Kopenawa et Bruce Albert, La Chute du Ciel. Paroles d'un chaman Yanomami, Paris, Plon, 2010, p. 99). Tout ce qui touche aux esprits est orné, brillant et dénué de substance : leurs chemins, les miroirs grâce auxquels ils se déplacent, les sols et les toits de leurs maisons, les clairières qu'ils aménagent dans la forêt. Ce sont de pures images qui se réfractent inlassablement les unes les autres. Les chants constituent eux-mêmes des « images » dont la particularité est de se fixer dans la pensée. «Les paroles des xapiri ne cessent de se rénover et ne peuvent être oubliées [...] elles s'accroissent et se fixent les unes après les autres à l'intérieur de nous et, ainsi, nous n'avons nul besoin de les dessiner pour nous en souvenir. Leur papier c'est notre pensée, devenue, depuis des temps très anciens, aussi longue qu'un grand livre interminable. » (Davi Kopenawa et Bruce Albert, La Chute du ciel. Paroles d'un chaman Yanomami, Paris, Plon, 2010 p. 554).

29. L'hypothèse émane d'une expérience de pensée appelée la "traduction radicale ", celle de la traduction du mot "Gavagaï » pour un locuteur d'une langue étrangère. Cette expérience de pensée démontre qu'il est possible de traduire de façons différentes un même énoncé d'un langage radicalement étranger. Ce qui doit être retenu, c'est que l'on obtient alors plusieurs traductions incompatibles entre elles, mais, pour autant, tout-à-fait correctes, en tant que traductions de la langue étrangère. De cette expérience de pensée émerge la thèse de l'inscrutabilité (ou indétermination) de la référence, qui jette un voile d'incompréhension entre deux locuteurs de langue différente. Il semble en effet que nous ne puissions jamais connaître ce que veut vraiment dire le locuteur d'une langue étrangère et que, à terme, nous ne puissions jamais saisir de quoi il parle. La relativité linguistique serait immanente (Willard Van Orman Quine, Word and Object, MIT Press, Cambridge [MA], 1960.)

30. Ces dards nommés wiruti en quechua occupent une position liminale entre l'intériorité et l'extériorité, puisqu'ils émanent à la fois concrètement du ventre, du corps ou de la gorge du chamane dont ils sont expulsés grâce à des techniques respiratoires spécifiques; et corollairement, ils sont considérés comme émanant du corps du patient: ce corps malade, victime d'une agression sorcellaire a été « pénétré » (yaykushka) par le principe pathogène venu s'y loger.

31. Entre autres : Banisteriopsis caapi, Psychotria Viridis et de multiples autres adjuvants (voir par exemple Luis Eduardo Luna, «Icaros: Magic Melodies Among the Mestizo Shamans of the Peruvian Amazon », in E. Jean Matteson Langdon and Gerhard Baer [dir.], Portals of Power: Shamanism in South America, Albuquerque, University of New Mexico Press, 1992).

32. Les techniques corporelles du chamane renvoient à un schème commun à la plupart des groupes du Nord-Ouest amazonien: le schème de l'ingestion et de l'extraction. Le chamane extrait par succions successives les fléchettes - matérialisation de la cause de la maladie - de l'intérieur du corps du patient.

33. Une partie de ce chant a été extraite afin de ne pas alourdir l'analyse. Elle décline l'apparence, le mouvement, de chaque esprit-animal tenu responsable de l'ensorcèlement: kamichi cornu, vautour chauve, tapir, etc. 


\section{RÉSUMÉS}

Cet article interroge à partir d'un exemple amazonien l'un des enjeux central de l'énonciation rituelle chamanique : l'opération de composition-traduction qui s'impose au spécialiste rituel pour « représenter » les invisibles au cours de sa performance, et les dispositifs sensoriels qu'elle implique. En effet, l'étude des chants thérapeutiques ikara des peuples Runa d'Amazonie péruvienne (Haut Pastaza) et du contexte dans lequel ils sont énoncés par le chamane yachak, met au jour le caractère énigmatique de la geste rituelle: la duplicité du signe, présence évanescente ou évocation secrète, image mentale qui persiste et se propage entre les participants. Par quels moyens linguistiques, gestuels ou sensoriels le chamane yachak parvient-il à résoudre le déficit de visibilité des êtres convoqués sur la scène des performances rituelles : esprits, démons, animaux ou entités hybrides? De quels outils l'anthropologie dispose-t-elle pour ausculter ce mouvement de contagion des images parmi les participants? Comment définir le dispositif sensoriel qui affecte le champ de la représentation et des croyances au cours du rituel? Cette contribution met en perspective l'approche lévi-straussienne de l'efficacité symbolique et l'approche pragmatique des rituels chamaniques amazoniens. Elle permet d'examiner l'hypothèse que c'est précisément, dans ce contexte, l'aspect lacunaire du signe - la transgression des règles de la communication ordinaire et le caractère partiel du déchiffrement - qui confère à l'image toute sa force. Le paradigme occidental de la communication, défini par les sciences du langage en termes d'intention signifiante ou de pertinence, se voit ici subverti au profit d'un modèle pragmatique fondé sur la multiplication des voix, des points de vue et des interprétations possibles de l'image.

\section{INDEX}

Mots-clés : Amazonie, chamanisme, communication rituelle, efficacité symbolique, Lévi-Strauss, pragmatique, sensorialité

\section{AUTEUR}

\section{ANDREA-LUZ GUTIERREZ CHOQUEVILCA}

Andrea-Luz Gutierrez Choquevilca occupe depuis 2012 la chaire Religions des Indiens SudAméricains : sociétés des basses terres à l'École pratique des hautes études. Maître de conférences à la $\mathrm{V}^{\mathrm{e}}$ section des sciences religieuses, elle est aussi membre du Laboratoire d'Anthropologie Sociale (UMR 7130, Collège de France) et affiliée au Laboratoire d'ethnologie et de sociologie comparative (Université Paris Nanterre). Ses recherches se fondent sur une ethnographie comparative des sociétés amérindiennes des basses terres, en particulier chez les Runa et Achuar du Pérou. Elles abordent le fait religieux et la transmission culturelle sous un éclairage nouveau, croisant les méthodes de l'anthropologie linguistique et de la pragmatique, de l'approche interactionnelle et de l'écologie. Ses publications portent sur la communication rituelle, les régimes de mémoire et relations entre oralité et écriture, la sociologie des interactions entre humains et non humains et l'ethnographie des façons quotidiennes d'apprendre et de transmettre le savoir parmi les traditions amérindiennes. Elle codirige notamment avec Pierre Déléage l'équipe anthropologie linguistique au Laboratoire d'anthropologie sociale, qui réunit anthropologues, sociologues, historiens et linguistes pour explorer ces thématiques. 